\title{
The effect of different tonsillectomy techniques on taste sensation in the early and late postoperative periods
}

\author{
Zeynep Nur Erolø, Bilge Türk®, Nurullah Seyhun $\odot$, Mahmut Cankaya $\odot$, Aytuğ Altundağ $\odot$, Berna \\ Uslu Coşkun®
}

Department of Otorhinolaryngology Head and Neck Surgery, Şişli Hamidiye Etfal Training and Research Hospital, Istanbul, Turkey

\begin{abstract}
Objectives: Impairment in taste sensation is a rare complication of tonsillectomy. We aimed to reveal the effects of different tonsillectomy techniques on taste sensation by assessing the impairment in taste sensation in the early and late postoperative periods.

Methods: In this prospective clinical study, fifty-seven (22 females, 35 males, mean age, $25 \pm 9.8$ years) patients who underwent tonsillectomy were included. All patients were operated on under general anesthesia, and a chemical stimulation taste test was performed one week prior to the surgery, at first week and first month postoperatively. Four basic taste sensations were assessed in the test (sweet, sour, salty, and bitter), and impairments in taste sensation in the early and late postoperative periods for each tonsillectomy technique were evaluated.

Results: In the early postoperative period, there was a statistically significant increase bitter taste sensations in patients who underwent tonsillectomy $(p=0.020)$. In the late postoperative period, sour and bitter taste sensations were significantly increased in the patients $(p=0.001, p=0.002$ respectively). In contrast to the early postoperative period, total taste sensation was significantly increased in the late postoperative period ( $p$ $=0.034)$.

Conclusions: The bitter sensation in the early postoperative period and the bitter and sour sensations in late postoperative period were found to be significantly increased in this present study. With regard to these results, a thorough preoperative explanation should be done.
\end{abstract}

Keywords: Tonsillectomy, taste sensation, chemogustometry

$\mathrm{T}$ onsillectomy is one of the oldest and most commonly performed surgery worldwide. Impairment in taste sensation after tonsillectomy is a rare complication, and it can affect quality of life in the postoperative period. Few studies reported on this complication, which is thought to be due to lingual nerve branch of the glossopharyngeal nerve traction during tonsillectomy or from indirect thermal injury
$[1,2]$. Impairment in taste sensation can lead to malnutrition, significant gains or losses in weight, and changes in dietary. To reduce the risk of complications of tonsillectomy which can cause significant morbidities, many techniques have been described, such as cold dissection, mono-polar and bipolar dissections, laser tonsillectomy cryosurgery, harmonic scalpel electro surgery, coblation, thermal welding, and

How to cite this article: Erol ZN, Türk B, Seyhun N, Cankaya M, Altundağ A, Uslu Coşkun B. The effect of different tonsillectomy techniques on taste sensation in the early and late postoperative periods. Eur Res J 2021;7(3):284-290. DOI: 10.18621/eurj.721469.

Address for correspondence: Zeynep Nur Erol, MD., Şişli Hamidiye Etfal Training and Research Hospital, Department of Otorhinolaryngology Head and Neck Surgery, İstanbul, Turkey.E-mail: drzeyneperol11@gmail.com 
plasma knife. The most frequently performed technique among these is cold dissection, but none of these techniques has been universally accepted.

Many studies compare the effects of various tonsillectomy techniques, but, to our knowledge, the effect of different techniques on taste sensation has not been investigated. In this study, we aimed to evaluate the effect of different techniques on taste sensation in the early and late postoperative periods following tonsillectomy.

\section{METHODS}

\section{Study Design and Population}

Fifty-seven (35 [61.4\%] males, 22 [38.6\%] females) patients with an age range of 12 to 50 (mean: $25 \pm 9.8$ ) years who underwent different tonsillectomy techniques were enrolled to this randomized and prospective study. Patients with chronic systemic diseases which could affect taste sensation (e.g., chronic kidney disease and middle ear diseases) and patients who were previously diagnosed with taste disorders, who were on medication, or who were operated on for bleeding as a complication of tonsillectomy were excluded from the study.

All patients were informed preoperatively about the study and surgical procedure, and written consent was obtained from each patient. The study protocol was approved by the local Ethics Committee (KAEK 2016/1145).

\section{Surgery Procedures}

Randomization of the groups to determine surgical technique was decided by lot. All patients were operated on under general anesthesia. The harmonic scalpel was used in $13(22.8 \%)$ patients, bipolar cautery was used in $18(31.6 \%)$ patients, cold dissection was used in $14(24.6 \%)$ patients, and thermal welding was used in $12(21.1 \%)$ patients. Preoperatively, $0.5 \mathrm{mg} / \mathrm{kg}$ of dexamethasone was administered intravenously to all patients (max. $8 \mathrm{~g}$ ). Oral feeding with cold liquids was started in patients without active bleeding or nausea/vomiting in the fourth postoperative hour. All patients were discharged on the first postoperative day.

Evaluation of Taste Sensation
Nowadays the two most commonly used tests are electrogustometry and chemogustometric test. Bitter, sour, sweet, salty and umami solutions are used in the chemical tests and electrical current is used in electrogustometry to create the sense of taste. Electrogustometry makes quantitative measurements whereas chemogustometry makes both quantitative and qualitative measurements [3]. For this reason, we used chemogustometric tests in our study.

Preoperative taste sensation was evaluated, and results were recorded one week prior to the surgery. Postoperative taste sensation was evaluated first week (early period) and first month (late period). The patients were informed about not eating, smoking or brushing their teeth for an hour before the test.

Four basic taste sensations were evaluated (sweet, sour, salty, and bitter) by using a chemical stimulation test. Filtered paper strips (Taste Strips; Burghart, Wedel, Germany) were used for the test. The strips were $8 \mathrm{~cm}$ long, with $2 \times 2 \mathrm{~cm}$ area of impregnated taste materials on the tip [3]. On the strips, four different concentrations for all four basic taste sensations were present. Distilled water was used as diluent and taste materials used, and the concentrations were as follows: (1) Sweet: 0.4, 0.2, 0.1, and 0.05 gr. sucrose $/ \mathrm{ml}$, (2) Sour: 0.3, 0.165, 0.09, and 0.05 gr. citric acid $/ \mathrm{ml}$, (3) Salty: $0.25,0.1,0.04$, and 0.016 gr. sodium chloride/ml, (4) Bitter: 0.006, 0.0024, 0.0009, and 0.0004 gr. kinin hydrochloride/ml.Taste solutions were freshly prepared at regular intervals. Strips were put on the left or right side of the tongue. Taste sensation is carried by chorda tympani nerve, which is a branch of facial nerve, on the anterior part of the tongue. Posterior part of the tongue is innervated by the lingual nerve which is a branch of glossopharyngeal nerve. Taste strips were applied to both parts of the tongue. Tonsillectomy can affect the taste sensation on the posterior part of tongue by damaging the lingual branch of glossopharyngeal nerve. However tonsillectomy does not cause any damage to chorda tympani which innervates the anterior part of the tongue. Therefore, our results reflects the effects of tonsillectomy on taste sensation of posterior part of tongue.

In total, 32 results were recorded. Prior to putting the strips on the tongue, patients were asked to rinse out their mouths. The test was done in increasing concentrations, and taste sensation was evaluated by randomly changing the sides for all four concentrations. 
Patients were asked to choose one of the four basic tastes for each strip. Taste scores were recorded for each side of the tongue for each concentration [4]. For each taste, both sides were analyzed statistically.

\section{Statistical Analysis}

SPSS 15.0 for Windows software (IBM, Armonk, NY, USA) was used for statistical analysis. Mean, standard deviation (SD), and median values were used in descriptive statistics. The one-way analysis of variation (ANOVA) and Kruskal Wallis test were used to compare independent variables. The Friedman test and the repeated measures ANOVA were used to compare more than two groups. P-values of 0.05 or less were considered significant.

\section{RESULTS}

Bitter taste sensation was found to be increased in the early postoperative period and was statistically significant ( $p=0.020)$ when evaluating the results, regardless of the technique used. In the late postoperative period, sour, bitter, and total taste sensations were increased and were also statistically significant ( $p=0.001, p=0.002, p=0.034$; respectively) (Table 1).

As it is defined, cold dissection is a cold knife technique, while hot dissection includes thermal welding, bipolar cautery, and harmonic scalpel techniques. In the early postoperative period, there was no statistically significant difference between the cold and hot technique groups in total taste sensation. However,

Table 1. Rate of taste changes in early and late postoperative period regardless of technique used

\begin{tabular}{llll}
\hline & & Mean \pm SD & p value \\
\hline Early period & & & \\
Sweet & Preoperative & $5.79 \pm 2.27$ & 0.425 \\
\hline Sour & Postoperative & $5.98 \pm 2.09$ & 0.107 \\
\hline Bitter & Preoperative & $4.04 \pm 2.28$ & $\mathbf{0 . 0 2 0}$ \\
\hline & Postoperative & $4.54 \pm 2.04$ & \\
\hline Salty & Preoperative & $4.42 \pm 2.91$ & 0.743 \\
\hline Total taste & Postoperative & $5.30 \pm 2.47$ & 0.133 \\
\hline Late period & Preoperative & $4.84 \pm 2.81$ & \\
\hline Sweet & Postoperative & $4.98 \pm 2.59$ & 0.061 \\
\hline Sour & Preoperative & $19.02 \pm 8.20$ & \\
\hline Bitter & Postoperative & $16.33 \pm 9.52$ & $\mathbf{0 . 0 0 1}$ \\
\hline Salty & Preoperative & $5.79 \pm 2.27$ & $\mathbf{0 . 0 0 2}$ \\
\hline Total taste & Postoperative & $6.32 \pm 1.89$ & \\
\hline & Preoperative & $4.04 \pm 2.28$ & 0.264 \\
\hline & Postoperative & $5.07 \pm 1.88$ & $\mathbf{0 . 0 3 4}$ \\
\hline & Preoperative & $4.42 \pm 2.91$ & \\
\hline & Postoperative & $5.47 \pm 2.44$ & $4.84 \pm 2.81$ \\
\hline
\end{tabular}

$\mathrm{SD}=$ standard deviation 


\begin{tabular}{|c|c|c|c|c|c|}
\hline & & \multicolumn{4}{|c|}{ Technique } \\
\hline & & \multicolumn{2}{|c|}{ Cold } & \multicolumn{2}{|c|}{ Hot } \\
\hline & & Mean \pm SD & Median & Mean \pm SD & Median \\
\hline \multicolumn{6}{|c|}{ Early period } \\
\hline \multirow[t]{3}{*}{ Sweet } & Preoperative & $6.1 \pm 2.0$ & 6.5 & $5.7 \pm 2.4$ & 6 \\
\hline & Postoperative & $5.9 \pm 1.9$ & 6 & $6.0 \pm 2.2$ & 6 \\
\hline & $p$ value & \multicolumn{2}{|c|}{0.633} & \multicolumn{2}{|c|}{0.280} \\
\hline \multirow[t]{3}{*}{ Sour } & Preoperative & $3.7 \pm 2.2$ & 4 & $4.1 \pm 2.3$ & 4 \\
\hline & Postoperative & $4.4 \pm 2.1$ & 4 & $4.6 \pm 2.0$ & 4 \\
\hline & $p$ value & \multicolumn{2}{|c|}{0.394} & \multicolumn{2}{|c|}{0.193} \\
\hline \multirow[t]{3}{*}{ Bitter } & Preoperative & $4.6 \pm 3.1$ & 4 & $4.4 \pm 2.9$ & 4 \\
\hline & Postoperative & $4.2 \pm 2.5$ & 5.5 & $5.7 \pm 2.4$ & 6 \\
\hline & $p$ value & \multicolumn{2}{|c|}{0.421} & \multicolumn{2}{|c|}{0.003} \\
\hline \multirow[t]{3}{*}{ Salty } & Preoperative & $5.0 \pm 3.2$ & 5.5 & $4.8 \pm 2.7$ & 4 \\
\hline & Postoperative & $4.9 \pm 3.2$ & 6 & $5.0 \pm 2.4$ & 5 \\
\hline & $p$ value & \multicolumn{2}{|c|}{0.680} & \multicolumn{2}{|c|}{0.655} \\
\hline \multirow[t]{3}{*}{ Total taste } & Preoperative & $19.4 \pm 8.6$ & 20.5 & $18.9 \pm 8.2$ & 20 \\
\hline & Postoperative & $15.7 \pm 9.8$ & 18.5 & $16.5 \pm 9.5$ & 19 \\
\hline & $p$ value & \multicolumn{2}{|c|}{0.135} & \multicolumn{2}{|c|}{0.299} \\
\hline
\end{tabular}

$\mathrm{SD}=$ standard deviation

when each taste sensation was evaluated, the postoperative bitter taste sensation was significantly increased compared to that of the preoperative period ( $p$ $=0.003$ ) (Table 2). In the late postoperative period, total taste, bitter taste, and sour taste were significantly increased in the hot technique groups compared to those in the preoperative period $(p=0.002, p=0.001$, $p=0.031$; respectively) (Table 3 ).

Posttonsillectomy bleeding and oral intake limitation were seen as complications, and complication rates for each technique were recorded; posttonsillectomy bleeding was observed in 7 patient, posttonsillectomy infection was observed in 3 patients but no statistically significant differences were found among the different tonsillectomy techniques.

\section{DISCUSSION}

Tonsillectomycarries a significant risk of morbidities and can even be fatal. The most common morbidi- ties of tonsillectomy are dehydration, oral feeding deficiency, bacteremia, otalgia, and bleeding. Taste disorders following tonsillectomy are a rare complication, but can significantly affect the patient's quality of life. Some reported studies exist in the literature about this complication $[1,2]$. Chronic and recurrent inflammation in the oral cavity and oropharynx can also significantly affect taste sensation [5].

Tonsils are innervated by two discrete cranial nerves: the lesser palatine branch of the maxillary nerve and the tonsillar branch of the glossopharyngeal nerve. The main innervation of the tonsillapalatina is by the tonsillar branch of the glossopharyngeal nerve. The lingual branch of the glossopharyngeal nerve, runs adjacent to the capsule of the tonsillapalatina in $21 \%$ of the adult population, and it can be injured during tonsillectomy, causing taste disorders on the posterior part of the tongue [4]. Inflammations in the tonsillar region can also cause otalgia via the tympanic branch of the glossopharyngeal nerve.

In the literature, it is reported that direct injury or 


\begin{tabular}{|c|c|c|c|c|c|}
\hline & & \multicolumn{4}{|c|}{ Technique } \\
\hline & & \multicolumn{2}{|c|}{ Cold } & \multicolumn{2}{|c|}{ Cold } \\
\hline & & Mean \pm SD & Median & Mean \pm SD & Median \\
\hline \multicolumn{6}{|l|}{ Late period } \\
\hline \multirow[t]{3}{*}{ Sweet } & Preoperative & $6.1 \pm 2.0$ & 6.5 & $5.7 \pm 2.4$ & 6 \\
\hline & Postoperative & $6.4 \pm 1.8$ & 7 & $6.3 \pm 1.9$ & 7 \\
\hline & $p$ value & \multicolumn{2}{|c|}{0.435} & \multicolumn{2}{|c|}{0.098} \\
\hline \multirow[t]{3}{*}{ Sour } & Preoperative & $3.7 \pm 2.2$ & 4 & $4.1 \pm 2.3$ & 4 \\
\hline & Postoperative & $4.7 \pm 2.0$ & 5 & $5.2 \pm 1.9$ & 5 \\
\hline & $p$ value & \multicolumn{2}{|c|}{0.242} & \multicolumn{2}{|c|}{0.002} \\
\hline \multirow[t]{3}{*}{ Bitter } & Preoperative & $4.6 \pm 3.1$ & 4 & $4.4 \pm 2.9$ & 4 \\
\hline & Postoperative & $4.2 \pm 2.6$ & 5.5 & $5.7 \pm 2.4$ & 6 \\
\hline & $p$ value & \multicolumn{2}{|c|}{0.748} & \multicolumn{2}{|c|}{0.001} \\
\hline \multirow[t]{3}{*}{ Salty } & Preoperative & $5.0 \pm 3.2$ & 5.5 & $4.8 \pm 2.7$ & 4 \\
\hline & Postoperative & $4.9 \pm 2.9$ & 6 & $5.3 \pm 2.1$ & 5 \\
\hline & $p$ value & \multicolumn{2}{|c|}{0.776} & \multicolumn{2}{|c|}{0.184} \\
\hline \multirow[t]{3}{*}{ Total taste } & Preoperative & $19.4 \pm 8.6$ & 20.5 & $18.9 \pm 8.2$ & 20 \\
\hline & Postoperative & $20.0 \pm 8.5$ & 21.5 & $21.1 \pm 8.4$ & 23 \\
\hline & $p$ value & \multicolumn{2}{|c|}{0.674} & \multicolumn{2}{|c|}{0.031} \\
\hline
\end{tabular}

$\mathrm{SD}=$ standard deviation

indirect thermal injury of the lingual branch of the glossopharyngeal nerve during tonsillectomy can also cause a significant decrease in taste sensation [3]. Anatomical variations of the lingual branch of the glossopharyngeal nerve play an important role in this. In $21 \%$ of the adult population, the lingual branch of the glossopharyngeal nerve runs adjacent to the capsule of the tonsillapalatina, which puts it at risk of injury [6].

In the present study, irrespective of which technique was used, bitter and sour taste sensations were significantly increased in the late postoperative period. Bitter taste sensation was also increased in the early postoperative period. Unlike the expectation of deterioration in the taste sensation, these results were notable. However, our findings did not match the literature regarding this point.

Taste sensation on the posterior part of the tongue is innervated by the lingual branch of the glossopharyngeal nerve; therefore, following tonsillectomy, it is expected to see taste changes in sour and bitter tastes
[7]. Previous reports on this mostly considered the taste sensation in the anterior part of the tongue, but the lingual nerve, a branch of the glossopharyngeal nerve, innervates the posterior part of the tongue [8]. However, in our study, contrary to the previous findings, bitter and sour taste sensations were significantly increased. This is thought to be the effect of inflammation of the oropharynx on taste sensation.

In a study by Stathas et al. [7], 60 patients who underwent cold knife and electrocautery tonsillectomy were evaluated for taste sensations with chemogustometry on the 1st, 15 th, and 30th postoperative days. On the first postoperative day, the posterior part of the tongue was found to be more affected than the anterior part of the tongue. Bitter and sour tastes were more affected than salty and sweet tastes. On the $30^{\text {th }}$ postoperative day, except for two patients, normal taste functions were regained [7]. This data shows that taste disorders following tonsillectomy are temporary. The authors suggest that this could be due to direct or indirect lingual branch of the glossopharyngeal nerve in- 
jury during the operation. Indirect injury can be due to compression of the mouth gag or local anesthetic infiltration.

In the literature, it is reported that lingual branch of the glossopharyngeal nerve injury is the main cause of taste disorders following tonsillectomy. During tonsillectomy, if the superior constrictor muscle cannot be conserved properly, lingual nerve injury may occur, and this can cause significant taste disorders [6]. In our study, regardless of the technique used, the patients did not experience any taste disorders postoperatively. This can be due to successful dissection during surgery, considering the first postoperative week as the early postoperative period, and a decrease in the chronic inflammation.

In one study, inflammation was found to affect taste sensation by toll-like receptors and type I-II interferons, which caused a decrease in taste sensations [5]. Our patients were 12 to 50 years of age and had experienced recurrent infections or chronic inflammation over a long time. In addition, the medications used for treatment, including anti-inflammatory and antibiotic medications, can also affect taste sensation [9]. Oral sprays that contain chlorhexidinegluconate are used for antiseptic purposes; they also can affect taste sensation [10]. All these findings are associated with the increase in taste sensation. The increase can also be related to decreased inflammation.

The increase in taste sensation in the harmonic scalpel, bipolar cautery, and thermal welding tonsillectomy groups, compared to cold techniques, is thought to be due to local injury which is more prevalent in cold techniques. The inflammation phase of wound healing occurs on days one to six. In this study, we tested the patients at the 1st week in the postoperative period, when the inflammation phase of wound healing was nearly over. At the 1st postoperative week, we believe, the effect of inflammation of taste sensation is at a minimum.

In this study, the results for the postoperative $1^{\text {st }}$ week and 30th day are similar, and this data is thought to be the result of the inflammation phase of wound healing which is usually over by the $6^{\text {th }}$ postoperative day. Irrespective of the techniques used, in the early postoperative period, bitter taste was significantly increased, and, in the late postoperative period, bitter, sour, and total taste sensations were significantly increased. These tastes are associated with the posterior part of the tongue, and this data can be explained by the decline in chronic inflammation, which has negative effects on taste sensation and shows that the lingual nerve was not injured during tonsillectomy.

A research was published on this subject in 2018. The highest prevalence of self-reported taste disturbances occurred two weeks after surgery (32\%). Two studies reported post-operative chemical gustometry scores consistent with hypogeusia. However, in the two studies that compared pre- and post-tonsillectomy test scores, one found no difference and the other found a significant difference only for the left rear of the tongue 14 days post-op. In the two studies that employed electrogustometry, elevated post-operative thresholds were noted, although only one compared pre- and post-operative thresholds. This study found no significant differences. No study employed a normal control group to assess the influences of repeated testing on the sensory measures. Overall, this review indicates that studies on post-tonsillectomy taste disorders are limited and ambiguous. [11].

\section{Limitations}

This study has a number of limitations. Small sample sizes and lengths of follow-up are the major limitations. However, the best of our knowledge, our analysis is the first study which evaluate taste disorders after different tonsillectomy techniques prospectively. When viewed from this aspect this study could flash on novel investigations. Further studies with larger cohorts should be conducted in evaluating the effects of tonsillectomy and its various surgical techniques on taste sensation.

\section{CONCLUSION}

In conclusion, although tonsillectomy may cause taste disorders as a rare complication, the bitter sensation in the early postoperative period and the bitter and sour sensations in late postoperative period were found to be significantly increased in the present study. With regard to these results, a thorough preoperative explanation should be done.

\section{Authors' Contribution}

Study Conception: ZNE, BT, MC; Study Design: ZNE, BT, AA; Supervision: ZNE, BT, BUC; Funding: 
ZNE, BT, AA; Materials: ZNE, NS, MC; Data Collection and/or Processing: ZNE, NS, MC; Statistical Analysis and/or Data Interpretation: ZNE, BT, MC; Literature Review: ZNE, BT, MC; Manuscript Preparation: ZNE, NS, MC and Critical Review: ZNE, NS, BUC.

\section{Conflict of interest}

The authors disclosed no conflict of interest during the preparation or publication of this manuscript.

\section{Financing}

The authors disclosed that they did not receive any grant during conduction or writing of this study.

\section{REFERENCES}

1. Tomita H, Ohtuka K. Taste disturbance after tonsillectomy. Acta Otolaryngol Suppl 2002;(546):164-72.

2. Heiser C, Landis BN, Giger R, Cao Van H, Guinand N, Hörmann $\mathrm{K}$, et al. Taste disorders after tonsillectomy: a long-term follow-up. Laryngoscope 2012;122:1265-6.

3. Mueller C, Kallert S, Renner B, Stiassny K, Temmel AFD, Hummel T, et al. Quantitative assessment of gustatory function in a clinical context using impregnated 'taste strips'. Rhinology 2003;41:2-6.

4. Philpott CM, Wild DC, Mehta D, Daniel M, Banerjee AR. A double-blinded randomized controlled trial of coblation versus conventional dissection tonsillectomy on post-operative symptoms. Clin Otolaryngol 2005;30:143-8.

5. Wang H, Zhou M, Brand J, Huang L. Inflammation and taste disorders: mechanisms in taste buds. Ann N Y Acad Sci 2009;1170:596-603.

6. Ohtsuka K, Tomita H, Murakami G. Anatomy of the tonsillar bed: topographical relationship between the palatine tonsil and the lingual branch of the glossopharyngeal nerve. Acta Otolaryngol Suppl 2002;(546):99-109.

7. Stathas T, Mallis A, Naxakis S, Mastronikolis NS, Gkiogkis $\mathrm{G}$, Xenoudakis D, et al. Taste function evaluation after tonsillectomy: a prospective study of 60 patients. Eur Arch Otorhinolaryngol 2010;267:1403-7.

8. Windfuhr JP, Schlondorff G, Sesterhenn AM, Kremer B. From the expert's office: localized neural lesions following tonsillectomy. Eur Arch Otorhinolaryngol 2009;266:1621-40.

9. Sakaguchi A, Nin T, Oka H, Maeda E, Negoro A, Umemoto $\mathrm{M}$, et al. [Clinical analysis of 1059 patients with taste disorders]. Nihon Jibiinkoka Gakkai Kaiho 2013;116:77-82. [Article in Japanese]

10. Gent JF, Frank ME, Hettinger TP. Taste confusions following chlorhexidine treatment. Cem Senses 2002;27:73-80.

11. Soldatova L, Doty Richard L. Post-tonsillectomy taste dysfunction: myth or reality? World J Otorhinolaryngol Head Neck Surg 2018;4:77-83. 